\title{
mbp-Seminare in Zusammenarbeit mit der DGOR 1984
}

Entwurf von EDV-Sollkonzepten

Zielgruppe: Qualifizierte Mitarbeiter aus EDV-Anwendungsabteilungen auch Sytemanalytiker und EDV-Projektleiter. Es werden keine spezifischen EDV-Kenntnisse vorausgesetzt.

Referent: Prof. Dr. H. Müller-Merbach, Universität Kaiserslautern

Termin u. $\quad$ 13./14. September 1984 in München

Ort:

Gebuhrren: DM 890,- zuzügl. MWST.

Rechnergestützte Entscheidungsunterstützungssysteme für das Management

Zielgruppe: Führungskräfte aus Planungs-, Organisations-, Marketing- und EDV-Abteilungen

Referent: Prof. Dr. H. Krallmann, TU Berlin

Termin u. 17. September 1984 in Berlin

Ort:

Gebühren: DM 530,- zuzügl. MWST.

\section{Petri-Netze}

Zielgruppe: Qualifizierte Mitarbeiter, die mit der Gestaltung und Analyse komplexer Systeme im Bereich Logistik, Daten- und Informationsverarbeitung, Organisation und Planung betraut sind.

Referenten: Dr. U. Winand, Universität Köln

Dr. W. Reisig, RWTH Aachen

Dr. B. Rosenstengel, Rheinische Olefin-Werke
Termin u. 25./26. Oktober 1984 in Dortmund

Ort:

Gebühren: DM 890,-zuzügl. MWST.

Stichprobeninventur in der Praxis

Zielgruppe: Qualifizierte Mitarbeiter aus Rechnungswesen, Revision, Lagerwirtschaft, Organisation und EDV-Anwendungsberatung sowie der Wirtschaftsprüfung.

Referenten: K.-P. Schuster, Philips GmbH, Hamburg Prof. Dr. V. Steinecke, HOESCH Werke AG, Dortmund

Termin u. $\quad$ 19./20. November 1984 in Hamburg

Ort:

Gebühren: DM 990,- zuzügl. MWST.

Prognoseverfahren in der Praxis

Zielgruppe: Qualifizierte Mitarbeiter aus Marketing/Marktforschung und -planung, Material- und Lagerwirtschaft

Referent: Prof. Dr. J. Schwarze, TU Braunschweig

Termin u. $\quad$ 10./11. September 1984 in München

Ort:

Gebühren: DM 890,- zuzügl. MWST.

Mathematischer Beratungs- und

Programmierdienst GmbH

Semerteichstraße 47,

4600 Dortmund 1 ,

Tel. (0231) 4348140

\section{Eingegangene Bücher}

Die im folgenden aufgelisteten Bücher sind zur Besprechung im OPERATIONS RESEARCH-SPEKTRUM eingegangen. Besprechungstexte zu einzelnen dieser Bücher werden in den folgenden Heften erscheinen.

Czogola, E.: Probabilistic Sets in Decision Making and Control. Köln: Verlag TƯV Rheinland, 1984, XIII, 113 Seiten, DM 36,-

Ellinger, Th.: Operations Research, Berlin/Heidelberg/New York/ Tokyo: Springer Verlag. 1984, X+269 Seiten, DM 39,-

Großeschallau, W.: Materialflußrechnung. Berlin, Heidelberg, New York, Tokyo: Springer Verlag, 1984, VIII, 222 Seiten, DM 42,-
Schaft, A. J. van der: System Theoretic Description of Physical Systems. Amsterdam: North-Holland, 1980, XV, 256 phages, hfl. 36,90

Tempelmeier, H.: Standortoptimierung in der Marketing-Logistik. Königstein. Anton Hain, 1980, IV, 266 Seiten, DM 89,-

$T u, P . \quad N . \quad V .:$ Introductory Optimization Dynamics. Berlin, Heidelberg, New York, Tokyo: Springer-Verlag, 1984, XIII, 387 pages, DM 39,- 